\title{
POSITIVE KNOTS AND LAGRANGIAN FILLABILITY
}

\author{
KYLE HAYDEN AND JOSHUA M. SABLOFF
}

(Communicated by Daniel Ruberman)

\begin{abstract}
This paper explores the relationship between the existence of an exact embedded Lagrangian filling for a Legendrian knot in the standard contact $\mathbb{R}^{3}$ and the hierarchy of positive, strongly quasi-positive, and quasipositive knots. On one hand, results of Eliashberg and especially Boileau and Orevkov show that every Legendrian knot with an exact, embedded Lagrangian filling is quasi-positive. On the other hand, we show that if a knot type is positive, then it has a Legendrian representative with an exact embedded Lagrangian filling. Further, we produce examples that show that strong quasi-positivity and fillability are independent conditions.
\end{abstract}

\section{INTRODUCTION}

Properly embedded Lagrangian submanifolds of $B^{4}$ whose boundaries are Legendrian links in $S^{3}$, called fillings of the Legendrian links, are of interest in a variety of fields: in smooth knot theory, Lagrangian fillings minimize the slice genus of a link [6]; in Legendrian knot theory, Lagrangian fillings induce augmentations of the Legendrian Contact Homology DGA [10 12] and Lagrangian fillings can even be used to answer questions about complex algebraic curves [5]. These considerations motivate the following question:

Which smooth knot types have Legendrian representatives with Lagrangian fillings?

Such a smooth knot type is termed fillable. In analyzing this question, we work in the equivalent setting of Legendrian links in the standard contact $\mathbb{R}^{3}$ and Lagrangian fillings in the symplectization $\mathbb{R} \times \mathbb{R}^{3}$. We further require the Lagrangian fillings to be exact, orientable, embedded, and collared, i.e. equal to $\mathbb{R} \times \Lambda$ outside a compact set.

Initial progress on the question above indicates a close relationship to the hierarchy of positivity in smooth knot theory. To describe the hierarchy, let $B P$ be the set of braid positive knot types, $P$ be the set of positive knot types, $S Q P$ be the set of strongly quasi-positive knot types, and $Q P$ be the set of quasi-positive knot types. The following relationships are well known (see [16, 22, 26, for example):

$$
B P \subsetneq P \subsetneq S Q P \subsetneq Q P .
$$

The first main result of this paper delineates a sufficient condition for a smooth knot type to be fillable:

Theorem 1.1. All positive knots are fillable.

Received by the editors August 1, 2013 and, in revised form, September 10, 2013.

2010 Mathematics Subject Classification. Primary 57R17, 57M25.

The second author was partially supported by NSF grant DMS-0909273. 
To make progress towards a necessary condition, we begin by noting that quasipositivity is necessary for fillability. To see this, first note that a result of Eliashberg [13] can be extended to show that a Lagrangian filling of a Legendrian knot may be perturbed to a symplectic filling of a transverse knot (see [5] for more details). From there, use the following result of Boileau and Orevkov [3]: if a smooth knot type has a transverse representative with a symplectic filling, then it is quasi-positive.

As we shall see in Section 4, however, not all quasi-positive knots are fillable. Further, the intermediate condition of strong quasi-positivity is independent of fillability:

Theorem 1.2. There exists a fillable knot that is quasi-positive, but not strongly quasi-positive, and there exists a non-fillable knot that is strongly quasi-positive.

Since this theorem shows that strong quasi-positivity is not relevant to fillability, we must seek an alternative condition to characterize fillable knots. Based on the results above and a survey of quasi-positive knots up to ten crossings 11 we make the following conjecture:

Conjecture 1.3. A smooth knot type $K$ is fillable if and only if it is quasi-positive and the HOMFLY bound on the maximum Thurston-Bennequin number of $K$ is sharp.

One implication of the conjecture is true: if a smooth knot is fillable, then, as discussed above, it is quasi-positive. Further, the Legendrian contact homology DGA of the fillable Legendrian representative has an augmentation [10,11, hence a graded ruling [15, 24, and hence the HOMFLY bound is sharp [23.

The remainder of the paper is organized as follows: after reviewing background on the various notions of positivity touched on above, on rulings of Legendrian knots, and on the construction of Lagrangian cobordisms via handle attachment in Section 2, we prove Theorem 1.1 in Section 3 and Theorem 1.2 in Section 4 .

\section{BACKGROUND}

In this section, we review positivity and (strong) quasi-positivity of smooth knots, rulings of Legendrian knots, and various constructions of Lagrangian fillings. We assume that the reader is familiar with basic notions of Legendrian knot theory as discussed, for example, in Etnyre's survey [14. In particular, we assume familiarity with front diagrams, the classical invariants, and the HOMFLY bound on the Thurston-Bennequin number.

2.1. Notions of positivity. The notion of positivity is simple to define: an oriented link is positive if it has a projection in which all crossings are positive. The jump to quasi-positivity requires the concept of positive bands in a braid, which generalize positive crossings. Denote the standard generators of the braid group by $\sigma_{i}$. A positive band is a braid word of the form $w \sigma_{i} w^{-1}$, where $w$ is any word in the braid group. The more restricted notion of a positive embedded band, denoted $\sigma_{i, j}$, is a positive band of the form

$$
\sigma_{i, j} \equiv\left(\sigma_{i} \cdots \sigma_{j-2}\right) \sigma_{j-1}\left(\sigma_{i} \cdots \sigma_{j-2}\right)^{-1}, \quad 1 \leq i<j \leq n .
$$

\footnotetext{
${ }^{1}$ We used [2] for a list of quasi-positive knots, Morrison's code in the KnotTheory package [1] and Morton and Short's C++ program [17]18] to compute the HOMFLY polynomials, Gridlink 8] to find Legendrian representatives of quasi-positive knots, and our own constructions of Lagrangian fillings.
} 

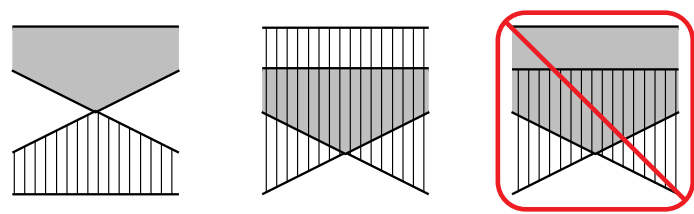

Figure 1. The two leftmost configurations of crossing and companion strands are allowed in a normal ruling (as is the reflection of the center configuration through a horizontal line), but the rightmost configuration is not allowed.

We define a (strongly) quasi-positive braid to be a product of positive (embedded) bands, and we say that an oriented link is (strongly) quasi-positive if it is the closure of such a braid.

The relationship between these notions of positivity - in particular, the inclusions displayed in equation (1.1) - has been treated extensively in the literature; see [16, 22, 26] for surveys.

2.2. Rulings of Legendrian links. A ruling is a combinatorial object associated to the front diagram of a Legendrian link. Hereafter, assume that all Legendrian links have been perturbed so that the cusps and crossings in their front diagrams have distinct $x$-coordinates. We define a ruling of such a front to be a one-to-one correspondence between left and right cusps, together with pairs of paths (called companions) in the front joining corresponding cusps. The companion paths must satisfy the following conditions:

(1) paired paths intersect only at the cusps they join; and

(2) unpaired paths intersect only at crossings.

In particular, the $x$-coordinate of each path is strictly monotonic and the paths cover the front. Further, since the companion paths meet only at the cusps, each pair of companions bounds a ruling disk in the plane.

To refine the notion of a ruling, we refer to the paths incident to a crossing of the front diagram as crossing paths. At a crossing, either the two crossing paths pass through each other or one path lies entirely above the other. In the latter case, we say that the ruling is switched at the crossing. We call a ruling normal if, at each switched crossing, the crossing paths have one of the configurations shown in Figure 1. We can rephrase this definition in terms of ruling disks: a ruling is normal if, near a switch, the interiors of the disks involved in the switch are either nested or disjoint. If all switches occur at positive crossings, then we say that the ruling is oriented 2

Example 2.1. The set of all oriented normal rulings of a Legendrian trefoil appear in Figure 2 .

The proof of Theorem 1.1 relies on rulings that switch at every crossing. In such a ruling, ruling disks are either (globally) nested or disjoint, and hence such a ruling must be normal. This type of ruling is equivalent to an "admissible 0resolution" of a front, as studied by $\mathrm{Ng}$ in [19]. A 0-resolution of a front is the diagram obtained by "smoothing" crossings as in Figure 3 . The aforementioned

\footnotetext{
${ }^{2}$ In the literature, such a ruling is also called 2-graded.
} 

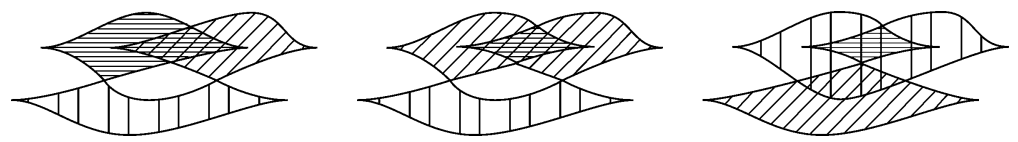

FIgURE 2. The three normal rulings of a Legendrian trefoil.

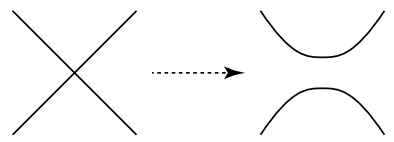

FiguRE 3. Smoothing a crossing using a 0-resolution.

paper shows that alternating links have Legendrian representatives with normal but not necessarily oriented - rulings that switch at each crossing. In Section 3 , below, we will revisit a result of Tanaka which implies that positive links also admit oriented normal rulings of this form.

2.3. Lagrangian handle attachment. In this subsection, we describe constructions for Lagrangian fillings of Legendrian links in the standard contact $\mathbb{R}^{3}$. A Lagrangian submanifold $L$ of the symplectization is a Lagrangian cobordism between the Legendrian submanifolds $\Lambda_{ \pm} \subset \mathbb{R}^{3}$ if there exists a pair of real numbers $T_{-}<T_{+}$such that

$$
\begin{aligned}
L \cap\left(\left(-\infty, T_{-}\right] \times \mathbb{R}^{3}\right) & =\left(-\infty, T_{-}\right] \times \Lambda_{-}, \text {and } \\
L \cap\left(\left[T_{+}, \infty\right) \times \mathbb{R}^{3}\right) & =\left[T_{+}, \infty\right) \times \Lambda_{+} .
\end{aligned}
$$

As defined in the introduction, a Lagrangian filling of a Legendrian link is a cobordism from the empty set to the given link. Note that stacking one Lagrangian cobordism on top of another results in a new Lagrangian cobordism.

The following tool allows us to construct Lagrangian fillings with a prescribed Legendrian link as their boundary. It has been adapted from Theorem 4.2 of [4], though this result appears in slightly different forms in the work of Ekholm-HondaKálman [12] and of Dimitroglou Rizell [9].

Theorem $2.2(4,6,9,12])$. If two Legendrian links $\Lambda_{-}$and $\Lambda_{+}$in the standard contact $\mathbb{R}^{3}$ are related by any of the following three moves, then there exists an exact, embedded, orientable, and collared Lagrangian cobordism from $\Lambda_{-}$to $\Lambda_{+}$.

Isotopy: $\Lambda_{-}$and $\Lambda_{+}$are Legendrian isotopic.

0-handle: The front of $\Lambda_{+}$is the same as that of $\Lambda_{-}$except for the addition of a disjoint Legendrian unknot as on the left side of Figure 4 .

1-handle: The fronts of $\Lambda_{ \pm}$are related as on the right side of Figure 4 ,

We will call a filling decomposable if it can be split into cobordisms arising as in Theorem 2.2. Hereafter, Theorem 2.2 will be our primary means for producing links with Lagrangian fillings.

\section{Positive Knots are fillable}

In this section, we prove Theorem 1.1. As mentioned above, the key tool in the proof is the existence of an oriented ruling in which all crossings are switched. The 

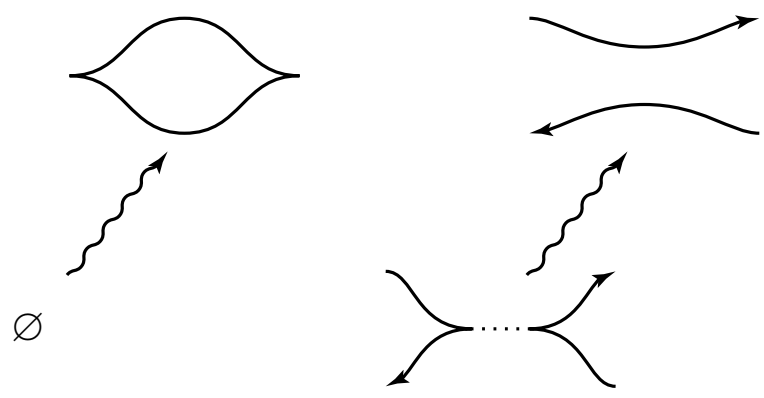

FIgURE 4. Diagram moves corresponding to attaching a 0-handle and an oriented 1-handle.

following lemma, essentially due to Tanaka [27, shows that we may assume the existence of such a ruling for a positive link.

Lemma 3.1. Every positive link admits a Legendrian representative whose front diagram carries an oriented normal ruling in which all crossings are switched.

Proof. In [27, Tanaka extends an earlier result of Yokota 28, to show that every positive link has a Legendrian representative $\Lambda$ with the following properties:

(1) Every crossing has both strands oriented to the left (in particular, every crossing is positive); and

(2) the 0-resolution of $\Lambda$ consists of disjoint (but possibly nested) closed curves, each of which contains exactly one left cusp and one right cusp.

By (11), the smoothing of each crossing in the 0-resolution respects the orientation of $\Lambda$. Thus, the strands which connect left and right cusps in the 0-resolution correspond to ruling paths in an oriented normal ruling that switches at each crossing.

We now prove that all positive links are fillable.

Proof of Theorem 1.1. By Lemma 3.1, it suffices to consider a fixed front $\Lambda_{+}$that admits an oriented normal ruling in which all crossings are switched. The proof proceeds by induction on the number of crossings. If $\Lambda_{+}$has no crossings, then it must be a disjoint union of maximal Thurston-Bennequin unknots. Such a link is fillable by the 0 -handle construction in Theorem 2.2

For the inductive step, suppose that every Legendrian link whose front diagram has fewer crossings than $\Lambda_{+}$and that admits an oriented normal ruling in which all crossings are switched is fillable. We begin by showing that the ruling of $\Lambda_{+}$must have a switch with a neighborhood equivalent to one of the topmost diagrams in Figure 5 (up to an overall reversal of orientation or reflection through the horizontal). First, each switch occurs at a positive crossing, so the crossing paths must have the same horizontal direction. Second, the companion paths to the crossing paths are oriented in the opposite direction to the crossing paths, so the two companion strands must also have the same horizontal direction. Finally, because the ruling switches at each crossing, the ruling disks are either nested or disjoint. Any switch along the boundary of an innermost ruling disk will have the desired form. 


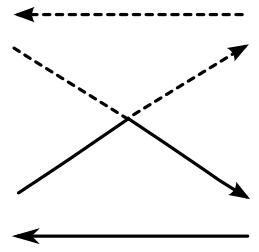

$\xi$

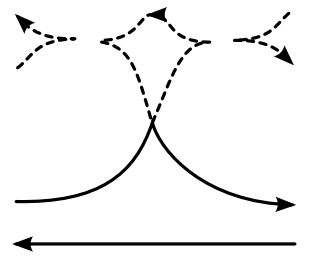

$\xi$
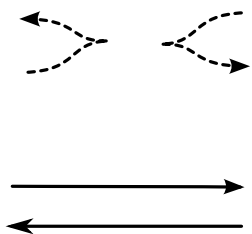

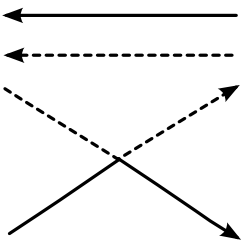

$\xi$

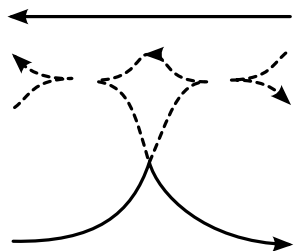

Isotopy
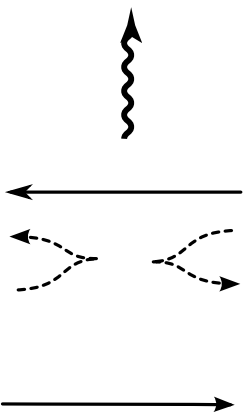

Figure 5. At the top are diagrams of $\Lambda_{+}$near the crossing constructed in the proof of Theorem 1.1] Diagrams of $\Lambda_{-}$appear at the bottom, and passing from bottom to top is a sequence of moves defining a Lagrangian cobordism from $\Lambda_{-}$up to $\Lambda_{+}$.

Next, we use the crossing found above to construct a Legendrian link $\Lambda_{-}$with the following properties:

(1) The fronts of $\Lambda_{+}$and $\Lambda_{-}$are identical outside of a neighborhood of the crossing;

(2) near the crossing, the front of $\Lambda_{-}$is of one of the forms depicted at the bottom of Figure 5 .

(3) $\Lambda_{-}$has an oriented normal ruling with all crossings switched; and

(4) there exists a Lagrangian cobordism from $\Lambda_{-}$to $\Lambda_{+}$.

Only the last condition needs some verification, which is carried out in Figure 5

Clearly, the Legendrian front $\Lambda_{-}$satisfies the inductive hypothesis and has one less crossing than $\Lambda_{+}$. It follows that $\Lambda_{-}$is fillable, and hence, by condition (4), that $\Lambda_{+}$is fillable as well. The theorem follows.

Remark 3.2. We say that an oriented ruling of a front has an unlinked resolution if the result of performing 0-resolutions (see Figure 3) at all switches of the ruling results in an unlink, all of whose components are maximal tb unknots. Using 


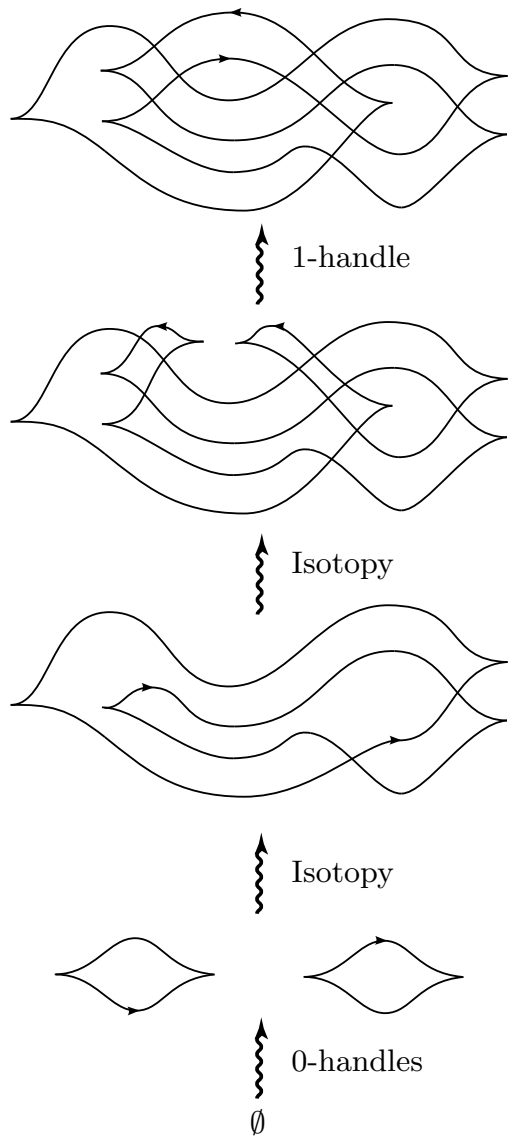

Figure 6 . The slice knot $m\left(9_{46}\right)$ is fillable.

this language, we see that the proof above actually gives a stronger result than Theorem 1.1 a smooth knot type is fillable if it has a Legendrian representative whose front has an oriented ruling with an unlinked resolution.

\section{4. (STRONG) QUASI-POSITIVITY AND FILLABILITY}

We end this paper by providing the examples necessary to prove Theorem 1.2 . The first example proves the first part of the theorem, namely that there exists a fillable quasi-positive knot that is not strongly quasi-positive.

Example 4.1. As shown in Figure 6, the mirror of the $9_{46}$ knot is fillable, and hence quasi-positive. On the other hand, since this knot is a non-trivial slice knot, its slice genus differs from its Seifert genus and hence it is not strongly quasi-positive [21, Prop. 2]. This example has appeared in [7] and [25], though in those papers, the Lagrangian filling is thought of as an example of a non-symmetric Lagrangian concordance between the $m\left(9_{46}\right)$ knot and the unknot.

The example for the second part of the theorem - that there exists a non-fillable strongly quasi-positive knot - is somewhat more complicated. It was originally 
brought to light by Stoimenow [26, Example 3] in the context of disproving a conjecture of Fiedler on an upper bound for the minimal degree of the Jones polynomial of a (quasi-positive) knot in terms of a band representation.

Example 4.2. Let $K$ be the closure of the strongly quasi-positive 4-braid

$$
\sigma_{1}^{2}\left(\sigma_{1} \sigma_{2} \sigma_{1}^{-1}\right) \sigma_{2} \sigma_{1} \sigma_{3}\left(\sigma_{1} \sigma_{2} \sigma_{1}^{-1}\right) \sigma_{2}\left(\sigma_{2} \sigma_{3} \sigma_{2}^{-1}\right)\left(\sigma_{1} \sigma_{2} \sigma_{1}^{-1}\right)\left(\sigma_{2} \sigma_{3} \sigma_{2}^{-1}\right)
$$

Suppose, for the sake of contradiction, that $K$ has a fillable Legendrian representative $\Lambda$. Denote the maximum Euler characteristic of a smooth slicing surface for $K$ by $\chi_{4}(K)$. We may easily compute from Rudolph's formula [20, Section 3] that $\chi_{4}(K)=-7$. Since $\Lambda$ is fillable, Theorem 1.3 of 6 implies that $t b(\Lambda)=-\chi_{4}(K)=7$ and that this is the maximal Thurston-Bennequin invariant for $K, \overline{t b}(K)$.

On the other hand, we comput $3^{3}$ that the degree in the framing variable of the HOMFLY polynomial of $K$ is -10 . Thus, the HOMFLY bound on the ThurstonBennequin number would be $\overline{t b}(K) \leq 9$. Clearly, this bound is not sharp. As argued after Conjecture 1.3. however, if $K$ were fillable, then the HOMFLY bound would be sharp. We must conclude, then, that $K$ is not fillable.

\section{ACKNOWLEDGEMENTS}

We thank Matt Hedden for several stimulating discussions about the material in this paper.

\section{REFERENCES}

[1] KnotTheory', Mathematica Package available at katlas.math.toronto.edu, accessed June 2013.

[2] Sebastian Baader, Slice and Gordian numbers of track knots, Osaka J. Math. 42 (2005), no. 1, 257-271. MR2132015 (2005m:57005)

[3] Michel Boileau and Stepan Orevkov, Quasi-positivité d'une courbe analytique dans une boule pseudo-convexe (French, with English and French summaries), C. R. Acad. Sci. Paris Sér. I Math. 332 (2001), no. 9, 825-830, DOI 10.1016/S0764-4442(01)01945-0. MR.1836094 (2002d:32039)

[4] F. Bourgeois, J. Sabloff, and L. Traynor, Lagrangian cobordisms via generating families: Constructions and Geography., Preprint available as arXiv:1409.3152, 2014.

[5] Chang Cao, Nathaniel Gallup, Kyle Hayden, and Joshua M. Sabloff, Topologically distinct Lagrangian and symplectic fillings, Math. Res. Lett. 21 (2014), no. 1, 85-99, DOI 10.4310/MRL.2014.v21.n1.a7. MR3247041

[6] Baptiste Chantraine, Lagrangian concordance of Legendrian knots, Algebr. Geom. Topol. 10 (2010), no. 1, 63-85, DOI 10.2140/agt.2010.10.63. MR2580429(2011f:57049)

[7] Baptiste Chantraine, Lagrangian concordance is not a symmetric relation, Preprint available as arXiv:1301.3767, 2013.

[8] M. Culler, Gridlink: a tool for knot theorists, Available at www.math.uic.edu/ culler /gridlink, accessed June 2013.

[9] G. Dimitroglou Rizell, Legendrian ambient surgery and Legendrian contact homology, Preprint available as arXiv:1205.5544v1, 2012.

$[10] \_$L Lifting pseudo-holomorphic polygons to the symplectisation of $P \times R$ and applications, Preprint available as arXiv:1305.1572, 2013.

[11] Tobias Ekholm, Rational SFT, linearized Legendrian contact homology, and Lagrangian Floer cohomology, Perspectives in analysis, geometry, and topology, Progr. Math., vol. 296, Birkhäuser/Springer, New York, 2012, pp. 109-145, DOI 10.1007/978-0-8176-8277-4_6. $\operatorname{MR} 2884034$

\footnotetext{
${ }^{3}$ Again, the computation was performed using Morrison's code in the KnotTheory package [1] and Morton and Short's C++ program [17].
} 
[12] T. Ekholm, K. Honda, and T. Kálmán, Legendrian knots and exact Lagrangian cobordisms, Preprint available as arXiv:1212.1519, 2012.

[13] Y. Eliashberg, Topology of 2-knots in $\mathbf{R}^{4}$ and symplectic geometry, The Floer memorial volume, Progr. Math., vol. 133, Birkhäuser, Basel, 1995, pp. 335-353. MR.1362834(96j:57023)

[14] John B. Etnyre, Legendrian and transversal knots, Handbook of knot theory, Elsevier B. V., Amsterdam, 2005, pp. 105-185, DOI 10.1016/B978-044451452-3/50004-6. MR2179261 (2006j:57050)

[15] Dmitry Fuchs and Tigran Ishkhanov, Invariants of Legendrian knots and decompositions of front diagrams (English, with English and Russian summaries), Mosc. Math. J. 4 (2004), no. 3, 707-717, 783. MR2119145 (2005i:57033)

[16] Matthew Hedden, Notions of positivity and the Ozsváth-Szabó concordance invariant, J. Knot Theory Ramifications 19 (2010), no. 5, 617-629, DOI 10.1142/S0218216510008017. $\operatorname{MR} 2646650(2011 \mathrm{j}: 57020)$

[17] H. R. Morton and H. B. Short, Homfly program, Available at http://www.liv.ac.uk/ su14 /knotprogs.html, accessed June 2013.

[18] H. R. Morton and H. B. Short, Calculating the 2-variable polynomial for knots presented as closed braids, J. Algorithms 11 (1990), no. 1, 117-131, DOI 10.1016/0196-6774(90)90033-B. MR:1041170 (91f:57004)

[19] Lenhard Ng, A Legendrian Thurston-Bennequin bound from Khovanov homology, Algebr. Geom. Topol. 5 (2005), 1637-1653, DOI 10.2140/agt.2005.5.1637. MR2186113(2007g:57027)

[20] Lee Rudolph, Quasipositivity as an obstruction to sliceness, Bull. Amer. Math. Soc. (N.S.) 29 (1993), no. 1, 51-59, DOI 10.1090/S0273-0979-1993-00397-5. MR1193540 (94d:57028)

[21] Lee Rudolph, An obstruction to sliceness via contact geometry and "classical" gauge theory, Invent. Math. 119 (1995), no. 1, 155-163, DOI 10.1007/BF01245177. MR.1309974 (95k:57013)

[22] Lee Rudolph, Knot theory of complex plane curves, Handbook of knot theory, Elsevier B. V., Amsterdam, 2005, pp. 349-427, DOI 10.1016/B978-044451452-3/50009-5. MR2179266 (2007b:57016)

[23] Dan Rutherford, Thurston-Bennequin number, Kauffman polynomial, and ruling invariants of a Legendrian link: the Fuchs conjecture and beyond, Int. Math. Res. Not., posted on 2006, Art. ID 78591, 15, DOI 10.1155/IMRN/2006/78591. MR2219227(2007a:57020)

[24] Joshua M. Sabloff, Augmentations and rulings of Legendrian knots, Int. Math. Res. Not. 19 (2005), 1157-1180, DOI 10.1155/IMRN.2005.1157. MR2147057 (2006e:57010)

[25] Steven Sivek, Monopole Floer homology and Legendrian knots, Geom. Topol. 16 (2012), no. 2, 751-779, DOI 10.2140/gt.2012.16.751. MR2928982

[26] A. Stoimenow, On polynomials and surfaces of variously positive links, J. Eur. Math. Soc. (JEMS) 7 (2005), no. 4, 477-509, DOI 10.4171/JEMS/36. MR2159224 (2006d:57014)

[27] Toshifumi Tanaka, Maximal Bennequin numbers and Kauffman polynomials of positive links, Proc. Amer. Math. Soc. 127 (1999), no. 11, 3427-3432, DOI 10.1090/S0002-9939-99-04983-7. MR.1616601 (2000b:57014)

[28] Yoshiyuki Yokota, Polynomial invariants of positive links, Topology 31 (1992), no. 4, 805811, DOI 10.1016/0040-9383(92)90011-6. MR1191382 (93k:57028)

Department of Mathematics, Boston College, Chestnut Hill, Massachusetts 02467

E-mail address: kyle.hayden@bc.edu

Department of Mathematics, Haverford College, Haverford, Pennsylvania 19041

E-mail address: jsabloff@haverford.edu 